\title{
The Surface Morphology and Microstructure of Ag ion-implanted Pyrolytic Carbon
}

\author{
Y. B. Chen, R. S. Zhou, K. D. Li, Q. M. Wei and Lu-Min Wang ${ }^{* * * *}$ \\ * Department of Nuclear Engineering \& Radiological Sciences, University of Michigan, Ann Arbor, \\ MI, 48109 \\ ** Department of Materials Science \& Engineering, University of Michigan, Ann Arbor, MI, 48109
}

Pyrolytic carbon $(\mathrm{PyC})$ has many potential applications due to its superior mechanical hardness, biocompatibility, as well as high thermal \& electric conductivities [1,2]. PyC has been proposed as one of the cladding materials for advanced nuclear fuel in the new generation of nuclear reactors. As a cladding material, PyC needs a strong mechanical robustness and high ability to retain fission products, such as silver, at elevated temperatures. In this work, we studied the effect of Agimplantation on the surface morphology and microstructure of PyC by electron microscopy.

Fig 1 (a)-(c) show the planar-view SEM images of PyC before implantation, PyC after implantation at ambient temperature and $400{ }^{\circ} \mathrm{C}$, respectively. One can see that $\mathrm{PyC}$ surface before implantation is quite smooth. But the surface of PyC after Ag-implantation is composed of near-periodic nanometer cellular as shown in Fig. 1(b)-(c). Statistical calculation revealed that carbon cellular are 100 $\mathrm{nm}$ in diameter and $1000 \mathrm{~nm}$ in height.

Fig. 2(a) is a high-angular annual dark-field (HAADF) scanning TEM image of PyC characterized in Fig. 1(c). It can be seen that many small Ag particles are agglomerated around the nanometer-sized cellular. Electron diffraction (not shown here) and high-resolution TEM corroborated that the Ag particles are crystalline. One of a typical HRTEM image was shown in Fig. 2(b). The corresponding fast-Fourier-transformation (FFT) image (upper-right inset) was indexed by [110] zone axis of facecentered cubic (FCC) metallic Ag. To study the change in chemical bonding after implantation, electron energy loss spectroscopy (EELS) was employed. Red and black curves in Fig. 2(c) show the carbon's core energy loss spectra ( $\pi^{*}$ and $\sigma^{*}$ peaks are labeled in figure) before and after Agimplantation, respectively. Compared these spectra with standard ones [3], it can be concluded that both of them show the amorphous carbon bonding features. However, spectrum of original PyC has a sharper $\pi^{*}$ peak compared with implanted one. According to standard method [4], it can be calculated that original $\mathrm{PyC}$ has higher $\mathrm{sp}^{2}$-type bonding percentage $(5-8 \%)$ than implanted one. Probably $\mathrm{sp}^{2}$ chemical bonds were broken by sputtering during Ag-implantation.

In conclusions, a smooth surface of PyC changes to a very rough one consisting of nanometer-sized cellular after Ag ion implantation. The implants form nanometer-sized Ag metal particles with FCC structure. Also, ion implantation decreases the percentage of $\mathrm{sp}^{2}$-type chemical bonding in PyC samples.

\section{References}

[1] S. D. Cook, et al., J. Bone Joint-Surg. Am. 81 (1999) 635.

[2] U. D. Abdelrazek, et al., J. Nucl. Mater. 249 (1997) 159.

[3] D. Shindo, et al., Analytical Electron Microscopy for Materials Science, Springer-Verlag, New York, 2002. 
[4] R. F. Egerton, Electron Energy-Loss Spectroscopy in the Electron Microscope, Plenum, New York, 1996.

[5] This research was supported by the DOE-NERI program.
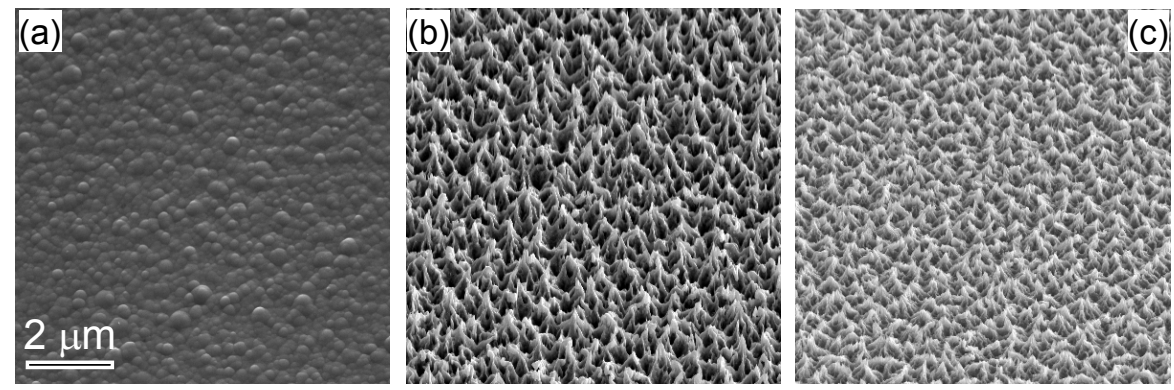

FIG. 1 Planar-view SEM images of original PyC (a), the PyC after Ag-implantation at ambient temperature (b) and $400{ }^{\circ} \mathrm{C}(\mathrm{c})$, respectively.
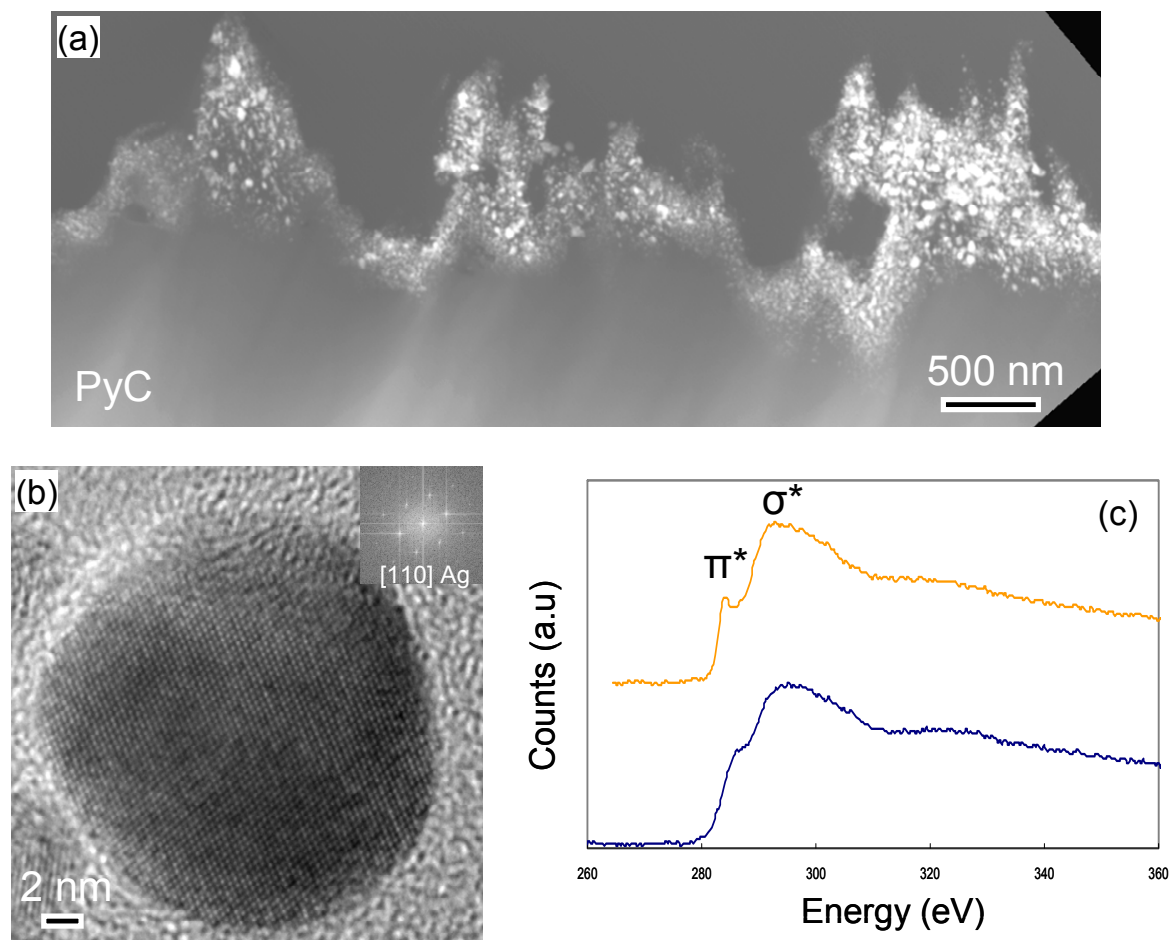

FIG. 2 (a) The HAADF micrograph of Ag-implanted PyC at $400^{\circ}$ C. (b) a HRTEM image of one Ag particle. Upper-right inset is the FFT image of the particle. (c) The EELS spectra of PyC before (red curve) and after Ag-implantation (black curve). 